\title{
Álgebra e Modelagem Matemática: um panorama das pesquisas brasileiras nos últimos anos
}

\author{
Algebra and Math Modeling: an overview of brazilian research in the last \\ years
}

\author{
Camilla do Valle Soares Cedraz \\ Vera Lucia Merlini \\ Eurivalda dos Santos Ribeiro Santana \\ Zulma Elizabete de Freitas Madruga
}

\begin{abstract}
Resumo: Nesse artigo, apresenta-se um panorama de estudos que envolvem Modelagem Matemática e Álgebra, voltados para a Educação Básica, com o objetivo de analisar como as pesquisas utilizam a Modelagem Matemática para auxiliar nas aulas de Álgebra. Para isso, foi realizada uma busca de dissertações e teses acerca dos temas citados, no Catálogo de Teses e Dissertações da Coordenação de Aperfeiçoamento de Pessoal de Nível Superior (CAPES), e a
\end{abstract} partir disso, foi realizado um refinamento e análise das pesquisas encontradas. Observou-se como esses trabalhos abordavam os conteúdos de Álgebra e de que maneira a Modelagem Matemática os auxiliou no processo. Como resultado, pode-se perceber que há pesquisas relacionando Modelagem Matemática com conteúdos de Álgebra, os quais em sua maioria abordam o conteúdo de funções com enfoque no Ensino Médio.

Palavras-chave: Modelagem Matemática. Álgebra. Panorama.

Abstract: In this article, a overview of studies involving Mathematical Modeling and Algebra, focused on Basic Education, is presented with the objective of analyzing how the researches use Mathematical Modeling to aid in Algebra classes. For that, a search of dissertations and theses on the themes was carried out, in the Catalog of Thesis and Dissertations of the Coordination of Improvement of Higher Level Personnel (CAPES), and from that, a refinement and analysis of the researches was carried out. It was observed how these works dealt with algebra contents and how mathematical modeling helped them in the process. As a result it can be noticed that there are researches relating Mathematical Modeling with algebra contents, and most of them approach the content of functions with focus in High School.

Keywords: Mathematical Modeling. Algebra. Overview.

Camilla do Valle Soares Cedraz

Mestranda em Educação Matemática pela Universidade Estadual de Santa Cruz (UESC). Bahia, Brasil. $\bowtie$ camillavs.cedraz@outlook.com

Vera Lucia Merlini iD Doutora em Educação Matemática (PUC-SP). Professora do Programa de Pós-Graduação em Educação Matemática pela Universidade Estadual de Santa Cruz (UESC). Bahia, Brasil. $\bowtie$ vera.merlini@gmail.com

Eurivalda dos Santos Ribeiro Santana Doutora em Educação Matemática (PUC-SP). Professora do Programa de Pós-Graduação em Educação Matemática pela Universidade Estadual de Santa Cruz (UESC). Bahia, Brasil. $\bowtie$ eurivalda@hotmail.com

Zulma Elizabete de Freitas Madruga

Doutora em Educação Matemática (PUC-SP) Professora Visitante do Programa de PósGraduação em Educação Matemática pela Universidade Estadual de Santa Cruz (UESC). Bahia, Brasil. $\square$ betefreitas.m@gmail.com

Recebido em 20/12/2018 Aceito em 02/02/2019 Publicado em 01/05/2019

\section{Introdução}

As práticas pedagógicas dos professores no cenário atual da educação precisam refletir as mudanças que estão acontecendo na sociedade, ou seja, no cotidiano dos estudantes. Tais 
práticas pedagógicas devem instrumentalizar os estudantes para a vida e para o trabalho, pois esses diariamente chegam ao ambiente escolar em busca de um conhecimento contextualizado com o cotidiano no qual estão inseridos. Em alguns casos, os professores não possuem 0 conhecimento de ferramentas que podem dar suporte às aulas, tornando-as atrativas e desafiadoras para esses estudantes. Como afirma Bassanezi (1994, p. 10), "grande parte do conhecimento matemático tem sido construído somente dentro do terreno da Matemática, a partir da ação de um profissional que em geral não formula questões como: Para que serve isso?

Sob esse enfoque, e para talvez responder a esse questionamento que a maioria dos estudantes fazem, supõe-se que a Modelagem Matemática seja um dos caminhos para amenizar tais dificuldades. Ressalta-se a importância de um trabalho contextualizado, respeitando 0 estudante e buscando desenvolver suas competências, habilidades e criatividade, tendo como suporte a Modelagem Matemática. Assim, este artigo tem como objetivo analisar como as pesquisas utilizam a Modelagem Matemática para auxiliar nas aulas que envolvem assuntos relacionados à Álgebra.

\section{Modelagem Matemática}

Relacionar os conteúdos matemáticos trabalhados em sala de aula com os contextos nos quais estão inseridos os estudantes é um desafio para a maior parte dos professores. Diante desse aspecto, o trabalho com Modelagem Matemática se faz presente e necessário na vida escolar. É importante proporcionar aos estudantes a oportunidade de se reconhecer como pesquisadores, buscando a resolução de problemas rotineiros. Nessa linha, aparece a Modelagem Matemática, a qual proporciona ao modelador fazer uma relação entre a Matemática e o mundo (BASSANEZI, 2010).

Para tanto, é necessário recorrer à construção de modelos que darão suporte a essas resoluções de problemas de uma maneira independente e própria, a este processo chama-se Modelagem. O modelo matemático consiste em um conjunto de representações matemáticas que buscam traduzir um problema do cotidiano. Biembengut e Hein (2003, p. 12) afirmam que o modelo matemático pode ser traduzido como "um conjunto de símbolos e relações matemáticas que procura traduzir, de alguma forma, um fenômeno em questão-problema de situação real".

O processo de Modelagem, segundo a concepção de Bassanezi (2010), começa com a escolha de um tema ou de uma situação-problema, a seguir há uma coleta de dados que podem 
ser qualitativos ou quantitativos, dependendo do tipo de dado que será coletado. Estes devem ser organizados em tabelas/quadros para que a análise seja mais eficiente. A partir disso, é possível obter uma melhor informação para a elaboração de um modelo matemático mais adequado para resolver o problema ou a situação-problema escolhida.

Em seguida, passa-se para a fase mais complexa e desafiadora do processo de Modelagem: a tradução da situação-problema para a linguagem matemática. Ao final dessa etapa, espera-se obter um conjunto de representações matemáticas que possibilitem uma resolução adequada para o problema em questão, ou seja, a formulação de um modelo matemático.

Depois da formulação do modelo matemático, inicia-se a resolução do problema em questão, para uma posterior validação e avaliação do modelo construído, com o intuito de verificar sua viabilidade de utilização. Nesse momento, inicia-se uma análise crítica das soluções encontradas, abrindo espaço para discussões. Este é apenas um dos 'modelos' do processo de modelagem, há autores que apresentam concepções diferentes que serão discutidas em trabalhos posteriores.

\title{
3 Procedimentos metodológicos
}

Este artigo apresenta uma pesquisa qualitativa, de acordo com Bogdan e Biklen (2010), e tem como princípio metodológico o mapeamento na pesquisa educacional, conforme Biembengut (2008). Para a autora, mapeamento é um

\begin{abstract}
princípio metodológico de pesquisa, significa principalmente, a compreensão da estrutura e dos entes nela inseridos, a organização e a representação ou mapa dos dados em um contexto, de forma dinâmica. Mapa que permita [...] explorar a habilidade da mente a buscar relacionar complexas imagens e, ao mesmo tempo, fornecer compreensão clara de um fenômeno ou ente em tempo curto de busca. (BIEMBENGUT, 2008, p. 2).
\end{abstract}

Para compor o mapeamento em questão foi realizada uma pesquisa bibliográfica em sites e catálogos virtuais, os quais continham documentos como teses e dissertações que abordavam o tema Modelagem Matemática, relacionados ao ensino de Álgebra para a Educação Básica. A intenção foi a de verificar trabalhos produzidos com essa temática na última década.

A investigação foi realizada no Catálogo de Teses e Dissertações da Coordenação de Aperfeiçoamento de Pessoal de Nível Superior (CAPES), buscando-se documentos que possuíam o termo "modelagem matemática" no título e/ou palavras-chave, para que, posteriormente, fosse 
avaliada quais delas possuíam relação com o ensino de conteúdos algébricos. Na primeira busca foram encontradas 4702 pesquisas; aplicando o filtro "ano", selecionando apenas as pesquisas dos últimos 10 anos, ou seja, entre 2008 e 2018, resultou em 3376 pesquisas. Utilizando o filtro "Grande área do conhecimento", nele selecionando as áreas Ciências Exatas e da Terra, Ciências Humanas e Multidisciplinar, o número de pesquisas reduziu para 1696. Filtrando em "área do conhecimento" Educação, Educação de Jovens e Adultos, Ensino, Ensino de Ciências e Matemática, Interdisciplinar e Matemática, resultou em 758 estudos. Refinando a pesquisa em "área de avaliação", optou-se por utilizar os filtros Educação, Ensino e Ensino de Ciências e Matemática, obtendo um quantitativo de 380 pesquisas. Em "área de concentração", selecionando Educação em Ciências e Matemática, Educação Matemática, Ensino de Ciências e Matemática, Ensino de Matemática e Ensino de Ciências e Educação Matemática, obteve-se 163 pesquisas.

Destas, foram analisados o título, as palavras-chave e os resumos de cada um dos trabalhos relacionados ao tema "Modelagem Matemática". Desses, 47 possuíam alguma relação com os conteúdos de Álgebra trabalhados na Educação Básica. Analisando com uma leitura dinâmica cada um deles, observou-se que apenas 20 relacionavam Modelagem Matemática (MM) com conteúdos de Álgebra na Educação Básica. Cabe dizer que os estudos restantes tratavam de pesquisas relacionadas ao Ensino Superior, o que foi descartado para este artigo. Dessa forma, para análise foram considerados os estudos organizados no Quadro 1.

Quadro 1: Pesquisas consideradas para análise

\begin{tabular}{|c|l|l|c|}
\hline Pesquisa & \multicolumn{1}{|c|}{ Título } & \multicolumn{1}{|c|}{ Autor } & Ano \\
\hline P1 & $\begin{array}{l}\text { Modelagem Matemática: um recurso facilitador no } \\
\text { processo ensino-aprendizagem }\end{array}$ & $\begin{array}{l}\text { Ângela Pereira } \\
\text { Baraldi }\end{array}$ & 2018 \\
\hline P2 & $\begin{array}{l}\text { Modelagem matemática: uma contribuição para a } \\
\text { construção do conhecimento matemático nos anos iniciais } \\
\text { da educação básica }\end{array}$ & Rodrigo Ruiz Brasil & 2013 \\
\hline P3 & $\begin{array}{l}\text { Estudo de função afim através da modelagem matemática } \\
\text { P4 }\end{array}$ & $\begin{array}{l}\text { A modelagem matemática como prática pedagógica no } \\
\text { ensino médio integrado em administração do IFSC - } \\
\text { Caçador }\end{array}$ & Flávio Fernandes \\
\hline P5 & $\begin{array}{l}\text { Progressões e funções: da variação e caracterização das } \\
\text { funções do tipo exponencial e logarítmica às técnicas de } \\
\text { ajuste de curvas no uso de modelagem matemática. }\end{array}$ & $\begin{array}{l}\text { Orlando Eduardo } \\
\text { Silva Ferri }\end{array}$ & 2014 \\
\hline
\end{tabular}




\begin{tabular}{|c|c|c|c|}
\hline P6 & O ensino de funções através de modelagem matemática & Eder Joacir de Lima & 2017 \\
\hline P7 & $\begin{array}{l}\text { A modelagem matemática como estratégia de preparação } \\
\text { para o ENEM }\end{array}$ & $\begin{array}{l}\text { Wilker Garcia } \\
\text { Magalhães }\end{array}$ & 2015 \\
\hline P8 & $\begin{array}{l}\text { Modelação matemática na sala de aula: o conceito de } \\
\text { função exponencial numa sequência de atividades para } 0 \\
1^{\circ} \text { ano do ensino médio }\end{array}$ & $\begin{array}{l}\text { Lina Flávia Morete } \\
\text { de Queirós Maia }\end{array}$ & 2017 \\
\hline P9 & $\begin{array}{l}\text { Modelagem matemática no estudo das funções afim e } \\
\text { quadrática. }\end{array}$ & $\begin{array}{l}\text { Alex Gonçalves de } \\
\text { Melo }\end{array}$ & 2017 \\
\hline P10 & $\begin{array}{l}\text { Modelagem matemática na escola básica: caracterização } \\
\text { do conhecimento matemático aprendido com Modelagem }\end{array}$ & $\begin{array}{l}\text { Patric Machado de } \\
\text { Menezes }\end{array}$ & 2017 \\
\hline P11 & $\begin{array}{l}\text { Investigando a Modelagem Matemática no ensino de } \\
\text { funções afins e exponenciais }\end{array}$ & $\begin{array}{l}\text { Ricardo Nogueira } \\
\text { Viana Narciso }\end{array}$ & 2016 \\
\hline P12 & $\begin{array}{l}\text { Uma proposta de ensino de matemática para a educação } \\
\text { básica }\end{array}$ & $\begin{array}{l}\text { Rodrigo Fernando } \\
\text { Nespolo }\end{array}$ & 2014 \\
\hline P13 & $\begin{array}{l}\text { O ensino e a aprendizagem de função exponencial em um } \\
\text { ambiente de modelagem matemática }\end{array}$ & $\begin{array}{l}\text { Antônio Josimário } \\
\text { Soares de Oliveira }\end{array}$ & 2013 \\
\hline P14 & $\begin{array}{l}\text { A abordagem da modelagem matemática em situações- } \\
\text { problema envolvendo funções afins e quadráticas no } \\
\text { ensino fundamental II }\end{array}$ & $\begin{array}{l}\text { Paulo Roberto } \\
\text { Figueiredo } \\
\text { Pamphylio }\end{array}$ & 2017 \\
\hline P15 & $\begin{array}{l}\text { Modelos matemáticos para a dinâmica populacional de } \\
\text { micro-organismos como motivação para o estudo de } \\
\text { potenciação, funções exponenciais e logarítmicas }\end{array}$ & $\begin{array}{l}\text { Carlos Eduardo } \\
\text { Bezerra dos Santos }\end{array}$ & 2015 \\
\hline P16 & $\begin{array}{l}\text { Progressões Geométricas e Música: Uma Proposta de } \\
\text { Modelagem }\end{array}$ & $\begin{array}{l}\text { Leniedson Guedes } \\
\text { dos Santos }\end{array}$ & 2014 \\
\hline P17 & Modelagem matemática na educação básica. & $\begin{array}{l}\text { Adilson Antônio } \\
\text { Sella }\end{array}$ & 2016 \\
\hline P18 & $\begin{array}{l}\text { A caracterização da função afim como ferramenta na } \\
\text { modelagem de problemas matemáticos. }\end{array}$ & $\begin{array}{l}\text { Francisco Eudes da } \\
\text { Silva }\end{array}$ & 2015 \\
\hline P19 & $\begin{array}{l}\text { Modelagem matemática no ensino médio: uma estratégia } \\
\text { para um aprendizado significativo }\end{array}$ & José Jociane Silva & 2018 \\
\hline P20 & $\begin{array}{l}\text { O uso da Modelagem Matemática no ensino de funções } \\
\text { na educação básica }\end{array}$ & $\begin{array}{l}\text { Sebastião } \\
\text { Rodrigues da Silva }\end{array}$ & 2014 \\
\hline
\end{tabular}

Fonte: Dados da Pesquisa 


\section{Resultados e discussão}

Com base na análise das 20 pesquisas selecionadas, pode-se perceber que essas abordam prioritariamente aplicações ou sugestões de aplicações de conteúdos algébricos com a Modelagem Matemática como ferramenta. Cinco desses trabalhos são voltados para turmas do Ensino Fundamental e 15 para turmas do Ensino Médio, sendo que um dos trabalhos possui uma proposta aplicada tanto no Ensino Fundamental quanto no Ensino Médio (P15). Dos 15 trabalhos voltados para o Ensino Médio, apenas uma pesquisa utilizou Progressões Geométricas como objeto matemático (P16), as demais utilizaram as funções, com exceção da P14 que utilizou-se das funções como objeto matemático, mas foi uma pesquisa voltada para os anos finais do Ensino Fundamental. Os dados estão organizados no Quadro 2.

Quadro 2: Conteúdos de Álgebra nas pesquisas mapeadas

\begin{tabular}{|l|c|}
\hline \multicolumn{1}{|c|}{ Conteúdo } & \multicolumn{1}{c|}{ Pesquisa } \\
\hline Decomposição de números & P2 \\
\hline Função & $\begin{array}{c}\text { P3; P4; P5; P6; P7; P8; P9; P10; P11; } \\
\text { P13; P14; P15; P17; P18; P19; P20 }\end{array}$ \\
\hline Equação & P1 \\
\hline Progressões geométricas & P16 \\
\hline $\begin{array}{l}\text { Números irracionais e operações com } \\
\text { números decimais }\end{array}$ & P12 \\
\hline
\end{tabular}

Fonte: Elaboração das Autoras

Além de utilizar a Modelagem Matemática (MM) como aporte para as aulas, em P2 (BRASIL, 2013), pode-se perceber que a abordagem do conteúdo decomposição de números, utilizou também uma metodologia intitulada "colar de contas". Em uma das etapas da pesquisa, aplicou-se uma oficina sobre MM utilizando essa metodologia para os professores da escola onde ocorreu a pesquisa. Tendo como base as dificuldades dos estudantes em aprender como decompor números naturais, o autor buscou, por meio da metodologia do colar de contas, desenvolver uma técnica que ajudasse tanto os professores quanto os alunos no processo de aprendizagem desse assunto. Tal metodologia utiliza colares feitos com cordão de nylon e contas que representam números naturais, conforme ilustra a Figura 1. 


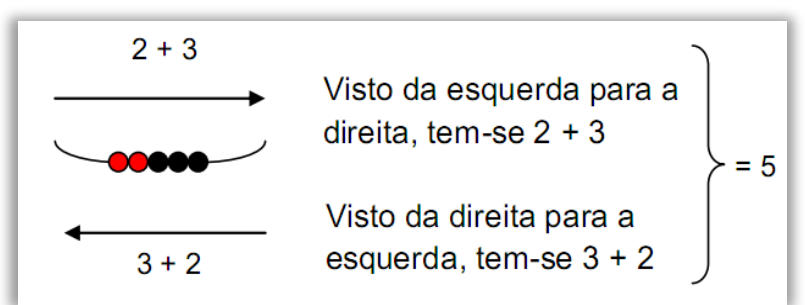

Figura 1: Metodologia "colar de contas" para a decomposição de um número natural (BRASIL, 2013, p. 33)

A partir dessa metodologia, o autor propõe também o trabalho com outros conteúdos como adição e multiplicação de números naturais e resolução de expressões numéricas. Segundo Brasil (2013), a aplicação da oficina apresentou um bom resultado no que diz respeito à utilização da Modelagem Matemática em sala de aula.

P1 (BARALDI, 2018), objetivou discutir a MM como um recurso para facilitar os processos de ensino e de aprendizagem de alunos dos anos finais do Ensino Fundamental, fazendo com que essa aprendizagem fosse significativa ao relacioná-la com situações da realidade do aluno, especificamente com relação ao ensino de equação. A autora aplicou uma atividade do livro Modelagem Matemática no Ensino (BIEMBENGUT e HEIN, 2003) em uma turma de Ensino Fundamental e outra de Ensino Médio, obtendo como resultado a incorporação dos conteúdos matemáticos de maneira significativa, mostrando, com isso, que atividades que utilizam a Modelagem Matemática conseguem despertar o interesse do aluno e fazer com que haja aprendizagem significativa.

Em P16 (SANTOS, 2014), propôs-se uma atividade voltada para alunos do Ensino Médio utilizando MM e uma relação entre Matemática e Música, abordando o conteúdo de progressões geométricas. Para isso, realizou-se um estudo do conceito de progressão geométrica e, em seguida, apresentou-se uma relação histórica entre conteúdos matemáticos e música. Por fim, o autor propôs uma atividade que envolveu o estudo de progressões geométricas e música a partir de uma oficina.

Em P12 (NESPOLO, 2014) desenvolveu-se uma atividade intitulada "Festa da Matemática", aplicada à uma turma de $6^{\circ}$ ano do Ensino Fundamental, abordando os conteúdos circunferência, números irracionais e operações com números decimais. Para isso, ele pesquisou sobre Modelagem Matemática como ferramenta metodológica em trabalhos publicados. Durante a aplicação da atividade, o autor pode perceber que os alunos se sentiram motivados em aprender 
os conteúdos matemáticos abordados, pois foram trabalhados de uma maneira que os conectavam com a realidade.

Do material encontrado que aborda o tema função ainda é possível subdividi-lo em função afim, função quadrática, função exponencial e logarítmica, conforme apresentado no Quadro 3.

Quadro 3: Tipos de funções encontradas nas pesquisas

\begin{tabular}{|l|l|}
\hline \multicolumn{1}{|c|}{ Tipo de função } & \multicolumn{1}{c|}{ Pesquisa } \\
\hline Função Afim & $\begin{array}{l}\text { P3; P4; P6; P7; P9; P10; P11; P14; P17; P18; } \\
\text { P19; P20 }\end{array}$ \\
\hline Função Quadrática & P6; P9; P14; P20 \\
\hline $\begin{array}{l}\text { Função Exponencial e } \\
\text { Logarítmica }\end{array}$ & P5; P6; P8; P11; P13; P15; \\
\hline
\end{tabular}

Fonte: Elaboração das Autoras

Observando as pesquisas que tratam sobre funções, optou-se por categorizá-las conforme os conteúdos de função apresentados: função afim, função quadrática e função exponencial e logarítmica.

Nas pesquisas analisadas que tratam da Modelagem Matemática utilizando a função afim como objeto matemático, foi possível observar que em P6, P10, P11 e P17 foram feitas aplicações de atividades envolvendo Modelagem e função afim para alunos do Ensino Médio. Dessas pesquisas, pode-se destacar P6, que compara o trabalho com esse conteúdo de uma maneira formal e o trabalho utilizando Modelagem, o qual obteve resultados satisfatórios com os estudantes. As demais pesquisas que utilizaram função afim como objeto matemático, fizeram sugestões de atividades que poderiam ser aplicadas em turmas do Ensino Médio.

Foi possível perceber que todas utilizaram temas diversificados, como a escolha de uma empresa de telefonia móvel (P3), Uber (P19) e comércio, viagens de táxi, distribuição de água, pintura de paredes e pagamento de estacionamentos (P9), evidenciando como a Modelagem permite contextualizar conteúdos matemáticos. Em P7 houve uma proposta com as questões do Exame Nacional do Ensino Médio (ENEM) como tema gerador para um trabalho diferenciado, o qual teve como objetivo motivar os alunos e fazer com que eles compreendessem os conteúdos abordados de uma maneira mais fácil. 
Por meio da análise dessas pesquisas, também foi possível perceber que há sempre uma crítica ao modelo tradicional de se trabalhar funções, crítica essa com o objetivo de trazer metodologias alternativas para o ensino do conteúdo, buscando suporte na Modelagem. A maior parte das pesquisas fundamenta-se nos autores Biembengut e Hein (2003); Bassanezi (1994, 2010); e Barbosa (2001).

As pesquisas P9, P14 e P20 propuseram sugestões de atividades utilizando como objeto matemático função quadrática, tendo como foco a obtenção de uma metodologia alternativa para o trabalho com esse conteúdo, sustentando-se na Modelagem. Apenas P6 aplicou uma atividade utilizando Modelagem e funções quadráticas em uma turma de Ensino Médio abordando a maximização de lucros como tema gerador.

Das pesquisas analisadas, P15 propôs 0 trabalho interdisciplinar com funções exponenciais e um conteúdo de Biologia, com sugestão de atividade para desenvolver um modelo matemático capaz de descrever o crescimento populacional de microrganismos. As pesquisas P6 e P11 fizeram uma aplicação com turmas do Ensino Médio, que segundo o autor de P11, obtevese resultados satisfatórios, por conseguir que os estudantes criassem um modelo matemático e, com ele, resolvessem problemas.

As demais pesquisas fizeram sugestões de atividades a serem trabalhadas com os estudantes envolvendo os conteúdos de função exponencial e função logarítmica. Em P8 houve uma busca em desenvolver as atividades de maneira que os alunos pudessem resolvê-las de maneira instintiva, para posteriormente formalizar o conteúdo matemático em questão. Em P13 há uma sugestão do trabalho com funções exponenciais e logarítmicas utilizando o software GeoGebra, por meio de uma atividade com o tema "cultura de bactérias" sugestionando uma possível interdisciplinaridade.

A análise das pesquisas indica que o trabalho com a Modelagem Matemática, relacionado aos conteúdos de Álgebra, motiva os alunos uma vez que rompe com o "afastamento" da Matemática e o cotidiano, proporciona trabalho interdisciplinar e estimula o estudante a pesquisar e desenvolver conhecimento lógico-matemático. 


\section{Considerações}

Este artigo teve como objetivo analisar como as pesquisas utilizam a Modelagem Matemática para auxiliar nas aulas que envolvem assuntos relacionados à Álgebra. Para tanto, foram analisadas 20 pesquisas que têm esses dois temas como foco de estudo.

Pode-se perceber que o trabalho com conteúdos algébricos, auxiliados pela Modelagem Matemática, está ganhando cada vez mais espaço nos últimos anos no ambiente de pesquisas científicas e, consequentemente, no ambiente escolar, algo que consideramos como favorável no que diz respeito à uma melhora na relação dos estudantes com a Matemática. Observando os resultados das aplicações de pesquisas feitas nesse viés, foi constatado que os estudantes pesquisados despertaram para aprender com mais facilidade, sendo motivados por um trabalho diferenciado ao abordar os conceitos algébricos de maneira contextualizada.

Além disso, pode-se notar diversas possibilidades de aplicações utilizando-se como recurso didático a Modelagem Matemática, dessa maneira possibilitando aos estudantes melhor compreensão dos conteúdos que envolvem Álgebra, tornando assim as aulas mais atrativas ao passo que o trabalho com Modelagem leva em consideração os saberes preexistentes dos estudantes além de fomentar discussões em sala de aula.

Nesse sentido, ressalta-se a importância de contextualizar os conteúdos, relacionando-os com o cotidiano dos estudantes, despertando assim o interesse pela Matemática presente no diadia. Como perspectiva de continuidade, tem-se a intenção de pesquisar mais trabalhos produzidos relacionando a Modelagem Matemática e outras áreas da Matemática, como Geometria, fazendo assim um mapa mais completo de pesquisas que têm a Modelagem Matemática como abordagem de estudo e discussão.

\section{Referências}

BARALDI, Ângela Pereira. Modelagem matemática: um recurso facilitador no processo ensinoaprendizagem. 2018. 101f. Dissertação (Mestrado em Matemática em Rede Nacional). Universidade Federal de Mato Grosso do Sul. Três Lagoas.

BARBOSA, Jonei Cerqueira. Modelagem Matemática: concepções e experiências de futuros professores. 2001. 253f. Tese (Doutorado em Educação Matemática) - Instituto de Geociências e Ciências Exatas. Universidade Estadual Paulista. Rio Claro. 
BASSANEZI, Rodney Carlos. Ensino-aprendizagem com Modelagem Matemática. São Paulo: Contexto, 2010.

BASSANEZI, Rodney Carlos. Modelagem como estratégia metodológica no ensino da Matemática. Boletim de Educação da SBMAC, São Paulo, 1994.

BIEMBENGUT, Maria Salett. Mapeamento como princípio metodológico na pesquisa educacional. Rio de Janeiro: Ciência Moderna, 2008.

BIEMBENGUT, Maria Salett; HEIN, Nelson. Modelagem matemática no ensino. 3. ed. São Paulo: Contexto, 2003.

BOGDAN, Roberto Carlos; BIKLEN, Sari Knopp. Investigação qualitativa em Educação. Tradução Maria João Alvarez, Sara Bahia dos Santos e Telmo Mourinho Baptista. Porto: Porto Editora, 2010.

BRASIL, Rodrigo Ruiz. Modelagem matemática: uma contribuição para a construção do conhecimento matemático nos anos iniciais da educação básica. 2013. 56f. Dissertação (Mestrado em Matemática em Rede Nacional). Universidade Federal de Rondônia. Porto Velho.

CAMELO, Soraya Martins. Estudo de função afim através da modelagem matemática. 2013. 49f. Dissertação (Mestrado em Matemática em Rede Nacional). Universidade Federal de Campina Grande. Campina Grande.

FERNANDES, Flávio. A modelagem matemática como prática pedagógica no Ensino Médio Integrado em Administração do IFSC - Caçador. 2016. 141f. Dissertação (Mestrado em Matemática em Rede Nacional). Universidade Federal da Fronteira Sul. Chapecó.

FERRI, Orlando Eduardo da Silva. Progressões e funções: da variação e caracterização das funções do tipo exponencial e logarítmica às técnicas de ajuste de curvas no uso de modelagem matemática. 2014. 67f. Dissertação (Mestrado em Matemática em Rede Nacional). Universidade Tecnológica Federal do Paraná. Curitiba.

LIMA, Eder Joacir de. 0 ensino de funções através de modelagem matemática. 2017. $88 \mathrm{f}$. Dissertação (Mestrado em Matemática em Rede Nacional). Universidade Federal de Mato Grosso. Barra do Garças.

MAGALHÃES, Wilker Garcia. A modelagem matemática como estratégia de preparação para 0 ENEM. 2015. 80f. Dissertação (Mestrado em Matemática em Rede Nacional). Universidade Federal de Mato Grosso do Sul. Campo Grande.

MAIA, Lina Flávia Morete de Queirós. Modelação matemática na sala de aula: o conceito de função exponencial numa sequência de atividades para $01^{\circ}$ ano do Ensino Médio. 2017. 68f. Dissertação (Mestrado em Matemática em Rede Nacional). Universidade Federal de São Carlos. Sorocaba

MELO, Alex Gonçalves de. Modelagem matemática no estudo das funções afim e quadrática. 2017. 67f. Dissertação (Mestrado em Matemática em Rede Nacional). Universidade Federal de Alagoas. Maceió. 
MENEZES, Patric Machado de. Modelagem matemática na escola básica: caracterização do conhecimento matemático aprendido com modelagem. 2017. 127f. Dissertação (Mestrado em Matemática em Rede Nacional). Universidade Federal da Fronteira Sul. Chapecó.

NARCISO, Ricardo Nogueira Viana. Investigando a Modelagem Matemática no ensino de funções afins e exponenciais. 2016. 84f. Dissertação (Mestrado em Matemática em Rede Nacional). Universidade Federal de Goiás. Catalão.

NESPOLO, Rodrigo Fernando. Uma proposta de ensino de Matemática para a educação básica. 2014. 49f. Dissertação (Mestrado em Matemática em Rede Nacional). Universidade Tecnológica Federal do Paraná. Pato Branco.

OLIVEIRA, Antônio Josimário Soares de. O ensino e a aprendizagem de função exponencial em um ambiente de modelagem matemática. 2013. 97f. Dissertação (Mestrado em Matemática em Rede Nacional). Universidade Federal Rural do Semi-Árido. Mossoró.

PAMPHYLIO, Paulo Roberto Figueiredo. A abordagem da modelagem matemática em situaçõesproblema envolvendo funções afins e quadráticas no Ensino Fundamental II. 2017. 65f. Dissertação (Mestrado em Matemática em Rede Nacional). Universidade Federal do Amapá. Macapá.

SANTOS, Carlos Eduardo Bezerra dos. Modelos matemáticos para a dinâmica populacional de micro-organismos como motivação para o estudo de potenciação, funções exponenciais e logarítmicas. 2015. 79f. Dissertação (Mestrado em Matemática em Rede Nacional). Universidade do Estado do Rio de Janeiro. Rio de Janeiro.

SANTOS, Leniedson Guedes dos. Progressões geométricas e Música: uma proposta de Modelagem. 2014. 63f. Dissertação (Mestrado em Matemática em Rede Nacional). Universidade Federal do Tocantins. Palmas.

SELLA, Adilson Antônio. Modelagem matemática na educação básica. 2016. 63f. Dissertação (Mestrado em Matemática em Rede Nacional). Universidade Federal de Mato Grosso. Cuiabá.

SILVA, Francisco Eudes da. A caracterização da função afim como ferramenta na modelagem de problemas matemáticos. 2015. 88f. Dissertação (Mestrado em Matemática em Rede Nacional). Universidade Federal do Ceará. Juazeiro do Norte.

SILVA, José Jociane. Modelagem matemática no ensino médio: uma estratégia para um aprendizado significativo. 2018. 75f. Dissertação (Mestrado em Matemática em Rede Nacional). Universidade Estadual do Piauí. Teresina.

SILVA, Sebastião Rodrigues da. O uso da modelagem matemática no ensino de funções na educação básica. 2014. 69f. Dissertação (Mestrado em Matemática em Rede Nacional). Universidade Federal do Amapá. Macapá. 